\title{
Signs of a new geomagnetic jerk between 2019 and 2020 from Swarm and observatory data
}

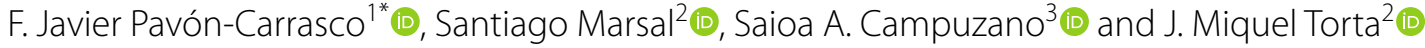

\begin{abstract}
Following the observed pattern of a new geomagnetic jerk every 3-4 years, certain predictions suggested that a new event should occur around 2020 after the one observed around 2017.5. In this work, we explore this scenario by analysing the secular variation of the East geomagnetic field component in both ground and satellite geomagnetic data. At ground, we use the available data from 2015 to 2021 in 10 observatories worldwide distributed. This analysis shows the occurrence of the mentioned jerk in mid-2017 at observatories located in the Pacific region, but also reveals a new jerk between mid-2019 and early 2020 with a clear global character. Swarm satellite data also corroborate these findings by means of the secular variation estimated using virtual observatories at $440 \mathrm{~km}$ altitude. In addition, a general view using the most recent CHAOS geomagnetic model confirms the global character of the 2020-jerk with V-shaped secular variation changes in meridional sectors covering the Eastern Pacific, America, Asia and the Indian Ocean; and $\Lambda$-shapes in Europe, Africa and Western Pacific. The radial geomagnetic field at the core-mantle boundary is investigated as the origin of the new jerk. Results show that the global-average secular acceleration of the radial field exhibits a new pulse at mid-2018, establishing the starting epoch of the 2020-jerk.
\end{abstract}

\section{Highlights}

- Observatory and Swarm data reveal a new geomagnetic jerk around 2019-2020.

- A very recent geomagnetic field model indicates the global character of the new jerk.

- A new pulse at the CMB around mid-2018 seems to be the starting point of this jerk.

Keywords: Geomagnetic jerks, Secular variation, Observatory data, Swarm data, Geomagnetic models

\section{Introduction}

Since late 2013, the European Space Agency's Swarm mission (Friis-Christensen et al. 2006) has provided accurate measurements of the geomagnetic field hundreds of kilometres above the Earth's surface. The three Swarm satellites, together with the continuous monitoring of the

\footnotetext{
*Correspondence: fjpavon@ucm.es

1 Universidad Complutense de Madrid (UCM), Plaza Ciencias, 1 ,

28040 Madrid, Spain

Full list of author information is available at the end of the article
}

ground observatories, allow the behaviour of the geomagnetic field to be described with unprecedented resolution. Among the numerous applications of this asset is the ability to refine the modelling of the geomagnetic main field, with implications for the dynamics of the Earth's outer core. In particular, we are interested in the characterization of abrupt changes in the trend of the geomagnetic secular variation (SV, the time derivative of the geomagnetic main field components), a phenomenon that has come to be known as geomagnetic jerk. 
The last two decades have been characterized by the occurrence of geomagnetic jerks in 2003 (Olsen and Mandea 2008), 2007 (Olsen et al. 2009), 2011 (Chulliat and Maus 2014), 2014 (Torta et al. 2015), and the most recent one in 2017, which was more regional in character than the preceding ones (Hammer 2018; Whaler et al. 2020). This succession of events points to a regular pace of about 3-4 years, suggesting that the jerks are caused by an oscillatory phenomenon within the outer core (Mandea et al. 2010; Chulliat and Maus 2014). In fact, recent studies (Kloss and Finlay 2019; Aubert and Finlay 2019) have indicated that the alternating equatorial bursts of flow acceleration, reflected in the radial geomagnetic field at the core-mantle boundary (CMB), are a marker for the occurrence of jerks. This phenomenon corresponds to the dynamics of the equatorial quasi-geostrophic (QG) Alfvén waves at the CMB that also modulates the variation in length of the day (LOD, Holme and De Viron 2013). This common geophysical source of jerks and LOD has been studied by Duan and Huang (2020), pointing out that the 8.6-year characteristic period of the LOD and the rate of 3-4 years for the occurrence of jerks are indeed correlated. Based on their results, these authors predicted the occurrence of a new geomagnetic jerk during 2020-2021.

A geomagnetic jerk is not observed everywhere at the same time and its amplitude depends on the location (Brown et al. 2013). The simplest way to detect a geomagnetic jerk at the Earth's surface is from the analysis of geomagnetic data recorded by ground-based magnetometers. Observatory practice involves an accurate revision of the data being processed to detect possible instrumental issues or artefacts that may need to be (re)checked. In this task, plots including the time evolution of the geomagnetic field components and its SV are useful to visually isolate problematic data. Using these plots, one can identify the possible occurrence of a jerk if the slope of the SV curve presents shape changes like $V$ or $\Lambda$. When this happens, an extended analysis including neighbouring observatories is carried out in order to decipher the regional or global character of the identified geomagnetic jerk. In addition, the ultimate support and confirmation of the occurrence of the jerk involves the use of satellite data, which is materialized by means of global geomagnetic field models. In terms of this kind of global modelling approach, the recent study of Wardinski et al. (2020) about a model candidate for the 13th International Geomagnetic Reference Field (IGRF-13, Alken et al. 2021) indicated the possible occurrence of two future geomagnetic jerks in late 2020-early 2021, and in early 2024.

Apart from the previous approach to identify the occurrence of jerks, there are other mathematical techniques for this purpose, such as the use of wavelets (Alexandrescu et al. 1996), Slepian functions (Kim and von Frese 2013) or nonlinear approaches (Qamili et al. 2013). Recently, Campuzano et al. (2021) have studied the occurrence of jerks using the characteristics of the South Atlantic Anomaly (SAA). They found a correlation between the occurrence of jerks and the minima of acceleration of the areal extent of the SAA. Their results confirm the jerks of the last years including that of 2017.

In this work, we have explored and validated the aforementioned prediction scenario for a jerk in 2020 by analysing the geomagnetic data available from groundbased observatories and Swarm satellites. At the ground level, 10 observatories worldwide distributed have been selected (not only because of geographical location, but also looking for the best data availability), and regarding the satellite data, we have used the Swarm-A spacecraft.

\section{Data and results}

\section{The 2020-jerk in geomagnetic observatory data}

In order to characterize the recent SV evolution, we have used data from 10 globally distributed INTERMAGNET (https://www.intermagnet.org) observatories (see locations in Figs. 3 and 4) for the last 6 years (from January 1st, 2015, to February 28th, 2021), for which the East component $(\mathrm{Y})$ has been employed assuming it is less sensitive to changes of external origin (especially magnetospheric). To further mitigate the influence of the external field, we have computed monthly mean values $\bar{Y}$ based on 1-min observatory data, either definitive, quasi-definitive or, exceptionally, provisional (in connection with this, the selection of the observatories was not only based on the uniformity of geographical locations, but also on the maturity of the available data). In addition, an estimate of the uncertainty $\Delta \bar{Y}$ of the monthly mean is obtained as the average standard deviation of the monthly means after detrending, where the latter is based on the double linear fit of the SV data described below. An alternative estimate of $\Delta \bar{Y}$ is obtained by the standard deviation of the daily mean values of the month divided by the square root of the number of days in that month. Both methods give results fairly consistent with each other, though we have used the first one in our calculations. With the computed $\bar{Y}$, the $\mathrm{SV}$ is calculated as the difference in times $t+6$ months and $t-6$ months (this is the standard way to estimate the SV, e.g. Chulliat et al. 2010), i.e. $\mathrm{SV}=\bar{Y}_{t+6}-\bar{Y}_{t-6}$, and its uncertainty is estimated as $\Delta \mathrm{SV}=\sqrt{\Delta \bar{Y}_{t+6}^{2}+\Delta \bar{Y}_{t-6}^{2}}$.

The above procedure has been applied to the geomagnetic $Y$ data of the 10 selected observatories. Results, in terms of SV and $\Delta S V$, are shown in Fig. 1 (see Additional file 1: Figure S1, where the monthly means are also plotted for each observatory). The obtained mean and SV values have been compared with the estimations from 


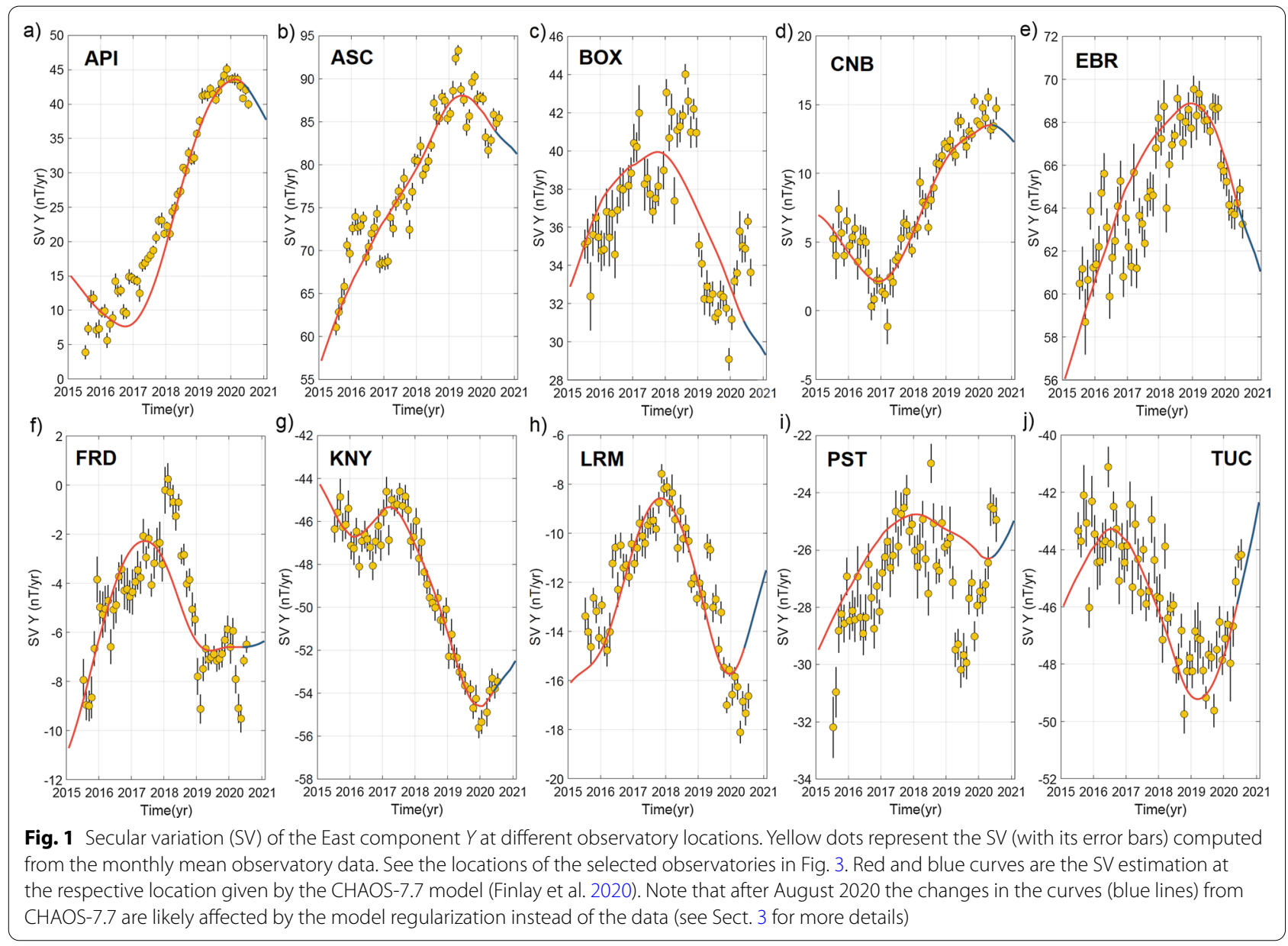

the CHAOS-7 model (Finlay et al. 2020, more information about this model is given in "Discussion: a global view from geomagnetic models" Section) for each observatory (red and blue lines in Fig. 1 and Additional file 1: Figure S1). Results show that most of the selected observatories have recorded the 2020-jerk around the nominal epoch of 2020, for which a V-shape is discernible at TUC, PST, BOX and KNY observatories, while a $\Lambda$-shape is observed at API, ASC and EBR. For these observatories, the SV curves from the CHAOS model agree except for BOX and PST, where the 2020-jerk is well-recorded by the data, but is not reflected in the geomagnetic model for BOX and with a clear delay (about 1 year) in PST. This could be because the SV in these observatories shows narrower $\mathrm{V}$-shapes than at the rest, making the CHAOS model predictions at these sites more difficult. At FRD, neither the data nor the model clearly show the jerk. Finally, at the location of LRM and CNB the CHAOS model shows the jerk at the beginning of 2020, though the SV data from these observatories do not record this event. In these latter cases, we think that more data covering the year 2021 are needed to constrain the jerk occurrence. It is worth noting that the SV of the CHAOS-7 model in epochs after mid-2020 can be strongly affected (edge effects) by some model parametrizations and thus it must be considered with caution (this issue is better detailed in "Discussion: a global view from geomagnetic models" Section). To remark this issue, the SV model estimations in Fig. 1 are plotted with another colour after mid-2020.

In order to obtain a global view of the characteristics of the 2020-jerk, we calculated its amplitude and occurrence time at different observatories covering a wide range of longitudes. To estimate these parameters, we follow the method of Pinheiro et al. (2011) based on the work of Le Mouël et al. (1982), which essentially consists in a double linear fit of the SV curve: one before the 2019/2020-jerk and another one after that time. Continuity of the two linear fits is imposed and the time of the jerk occurrence is also adjusted so that the $\mathrm{V}$ or $\Lambda$ shape minimizes the residuals with respect to the monthly SV data of Fig. 1 in the least squares sense (see the Additional file 1 for more details). The amplitude of the jerk at each observatory is taken as the difference of slopes before and after the jerk 
Table 1 Jerk occurrence time and amplitude at several observatories regarding the $Y$-component

\begin{tabular}{|c|c|c|c|c|}
\hline & \multicolumn{2}{|c|}{2017 nominal jerk } & \multicolumn{2}{|c|}{2020 nominal jerk } \\
\hline & Date (year) & Amplitude (nT/year $\left.{ }^{2}\right)$ & Date (year) & Amplitude (nT/year $\left.{ }^{2}\right)$ \\
\hline API & - & - & $2019.77 \pm 0.06$ & $-18 \pm 2$ \\
\hline TUC & - & - & $2019.70 \pm 0.08$ & $7.3 \pm 1.0$ \\
\hline ASC & - & - & $2019.21 \pm 0.15$ & $-13.3 \pm 1.2$ \\
\hline EBR & - & - & $2019.34 \pm 0.12$ & $-7.2 \pm 1.0$ \\
\hline LRM & $2018.05 \pm 0.08$ & $-6.0 \pm 0.4$ & - & - \\
\hline KNY & - & - & $2019.97 \pm 0.06$ & $9 \pm 2$ \\
\hline CNB & $2017.20 \pm 0.02$ & $6.7 \pm 0.4$ & - & - \\
\hline
\end{tabular}

Positive amplitudes denote $V$-shapes, while negative amplitudes denote $\Lambda$-shapes of the SV trend. The "-" symbol indicates that the jerk was not evident at that observatory or not enough data are available after the 2020-jerk

occurrence time. Additional file 1: Figure S2 shows the fitted SV data at the selected observatories. Table 1 shows the results and the associated uncertainties arising from the error propagation of $\Delta S V$. It should be noted, however, that the true uncertainty of the parameters of the 2020jerk is somewhat higher than that reported in the table due to the scarcity of data after the jerk as of the time of drafting this manuscript, thus preventing a robust fit. A clear example of this is the incipient change of slope guessed at LRM observatory around mid-2020 (Fig. 1), which could be the manifestation of the 2020-jerk in the region; however, in this case we have chosen not to include the fit due to the lack of data after the minimum. The analysis for the 2017-jerk has been included in the table for completeness, though its occurrence is not clear in most locations, even for API in the Pacific at ground level (see Fig. 1).

\section{The 2020-jerk in Swarm data}

To analyse how the 2020-jerk has been recorded by Swarm data, we have used all the satellite data available for the Swarm-A satellite from January 1st, 2015, to February 28th, 2021, i.e. the same time window as for observatories (note that any of the three Swarm satellites could have been chosen interchangeably for this purpose). Its dataset has been downloaded from the FTP server of ESA (ftp://Swarm-diss.eo.esa.int) and it corresponds to the Level1b product baseline 05 and file counter 05 (Olsen et al. 2013) that contains daily measurements of the geomagnetic field at $1-\mathrm{Hz}$ sampling rate (folders Entire_mission_data/MAGx_LR/Sat_A in the cited FTP server). Within the target time window, Swarm-A has measured the geomagnetic field between 430 and $470 \mathrm{~km}$ altitude, moving at a speed of about $7 \mathrm{~km} / \mathrm{s}$. Contrary to its excellent global coverage, the satellite movement makes it difficult to extract local mean values (monthly in our case) with the quality given by the observatory data, where the geomagnetic field is recorded at a fixed location. To solve this handicap when calculating the SV of the Y-component, we have treated the Swarm-A data following the modelling concept of the Geomagnetic Virtual Observatories (GVO, Mandea and Olsen 2006; Olsen and Mandea 2007; Hammer et al. 2021).

For each selected observatory location, we have first extracted the Swarm-A data in a spherical region centred at $440 \mathrm{~km}$ altitude over the observatory location with a radius of $250 \mathrm{~km}$. All the $S w a r m$-A satellite tracks within that 3D-region provide values of the $Y$-component at the satellite coordinates as a function of time. Secondly, in order to mitigate the effect of the external field contributions, we have applied the classical cleaning approach, which consists in keeping satellite data from dark regions and using some geomagnetic indices (Dst and $\mathrm{a}_{\mathrm{p}}$ ) to remove time periods of high external geomagnetic activity (see the Additional file 1 for more details about the applied filters). Thirdly, the CHAOS-7.7 model is also used to remove possible outliers and high data dispersion, comparing the filtered Swarm data and the model estimations. Finally, to get a time-continuous series of the Y-component at the fixed location of the GVO we have relocated all the data to the centre of the spherical region. To do that, we have shifted the satellite data to a common location according to the 3D-gradient provided by the CHAOS-7.7 model. Then, the SV is computed using overlapping windows 1.5 years in width moved every month from 2015 to 2021 . The slope of a linear fit applied within each time window gives the SV. The SV uncertainty is also obtained by the error of the linear fit. We are aware that the GVO processing algorithm recently developed by Hammer et al. (2021) is a more sophisticated approach, but our technique is enough to elucidate the main characteristics of the SV of the Y-component during the last 6 years. Results from some representative observatory locations at $440 \mathrm{~km}$ altitude are shown in Fig. 2 (see Additional file 1: Figure S3 for the rest of observatory locations). 


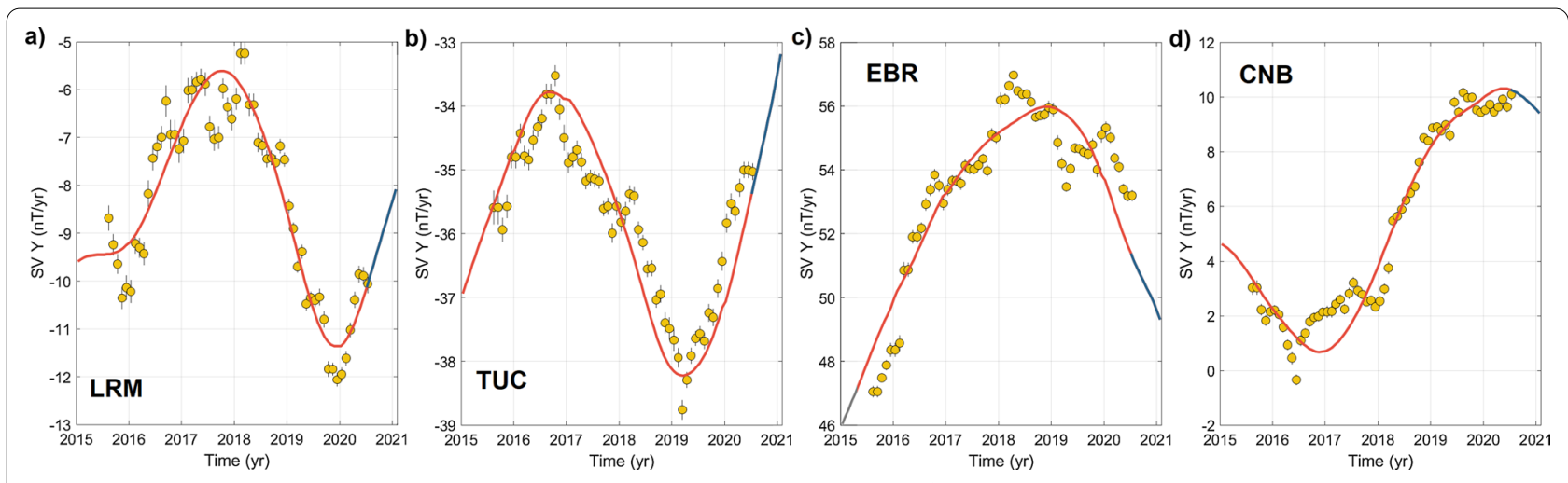

Fig. 2 Yellow dots: SV and its uncertainty (vertical black lines) of the East component $Y$ from Swarm-A data relocated at $440 \mathrm{~km}$ altitude over the indicated observatory locations (see main text for details). Red/blue curves: SV estimation from CHAOS-7.7 model. Note that after August 2020 the changes in the curves (blue lines) from CHAOS-7.7 are likely affected by the model regularization instead of the data (see Sect. 3 for more details)

These results confirm the previous observations at ground level. The 2020-jerk is clearly recorded by the Swarm-A data and is corroborated by the predictions of the CHAOS-7.7 model calculated at the centre of each spherical region. It is worth noting that the jerk is not recorded at LRM in terms of the SV data at ground level (see Fig. 1), however, the SV computed from the SwarmA data over this observatory shows the jerk around 2020.0. This fact reinforces our previous comment about the lack of availability of observatory data during 2021 (at the time of writing this work) to constrain the 2020-jerk in this observatory. In addition, caution must be taken after 2020.5 in the SV curves given by the CHAOS model due to edge effects (see "Discussion: a global view from geomagnetic models" Section for more details).

\section{Discussion: a global view from geomagnetic models}

To further investigate the evidence of this new jerk around 2020, we have used what is perhaps the most recent spherical harmonic model of the main geomagnetic field based on observatory and satellite data: the 7th release of the CHAOS-7 model (Finlay et al. 2020). This geomagnetic model is developed using Swarm satellite data up to 6th March 2021 and the available ground observatory data up to February 2021 (new releases of CHAOS can be found at https://www.space.dtu.dk). Although CHAOS-7 provides information about the geomagnetic main field (up to harmonic degree 15), the crustal field (up to harmonic degree 110) and the external field contributions, in this study, we just used its main field contribution. It should be noted that the CHAOS7.7 model has some limitations after August 2020. The model SV is strongly affected by the model regularization instead of the data and this affects the SV estimations during the final period of the occurrence of the 2020-jerk.
As a consequence, the SV changes (i.e. the SA) after mid2020 must be taken with caution along the present work. However, this fact does not affect our findings about the occurrence of a new jerk during 2019-2020.

To have a global picture of the occurrence of the 2020jerk, in Fig. 3a we have plotted the SV of the Y-component in 250 locations homogeneously distributed (following an equal area distribution) over the Earth's surface from January 1st, 2015, to February 28th, 2021. To complement this figure, we have drawn up Hovmöller diagrams across three latitudinal profiles (Fig. 3b). This type of diagram shows in the same panel the spatial and temporal evolution of a variable along the selected profile. Instead of plotting the SV values in the Hovmöllers, we have displayed the trend of the SV in terms of the sign of its time derivative, i.e. the sign of the secular acceleration (SA). Positive SA (red colour in Fig. 3b) indicates periods of increasing SV, while a negative SA (blue colour) represents periods of decreasing SV. To highlight the change of sign of the SA, i.e. the maximum or minimum of the SV that identifies the occurrence of a jerk, $\mathrm{SA}$ values close to $0 \mathrm{nT} /$ year $^{2}$ are highlighted in white. To point the utility of this plot, we give an example: for a location at $100^{\circ} \mathrm{W}$ longitude on the Earth's equator, the SA is positive from 2015 to 2017 when the 2017-jerk occurred (white colour). After that time, the SA turns negative up to the beginning of 2019 when the new 2020jerk is observed in that location and, since then, the SA is characterized by positive values (red colour). Note that, for a given timeline, the change of sign of the SA from positive to negative (i.e. from red to blue) corresponds to $\Lambda$-shaped jerks, while that from negative to positive (i.e. from blue to red) identify V-shaped jerks.

Considering the time window covering the last 6 years and as pointed out in the previous example, results from Fig. 3 do not only show the occurrence of 
(a)

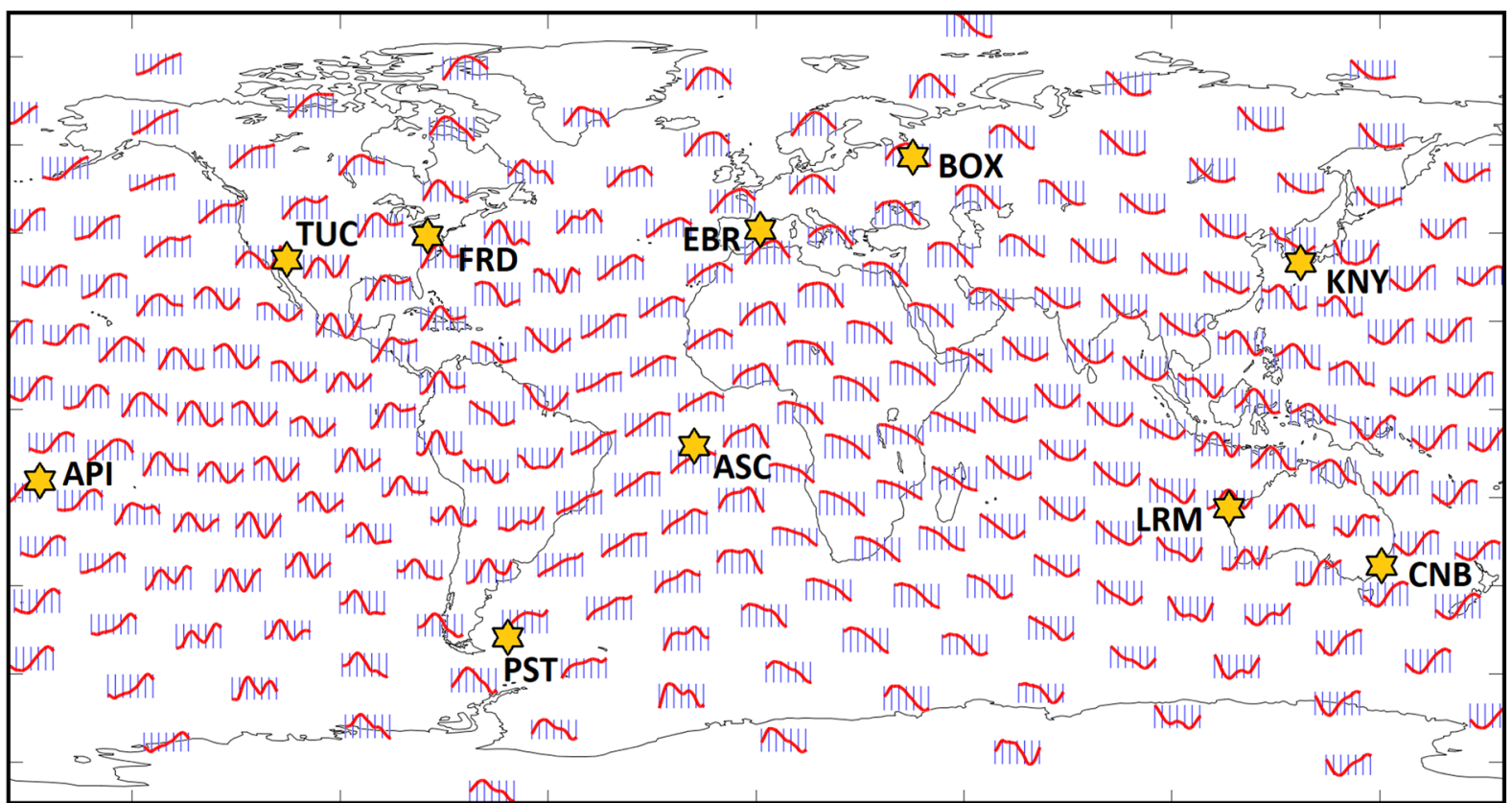

(b)
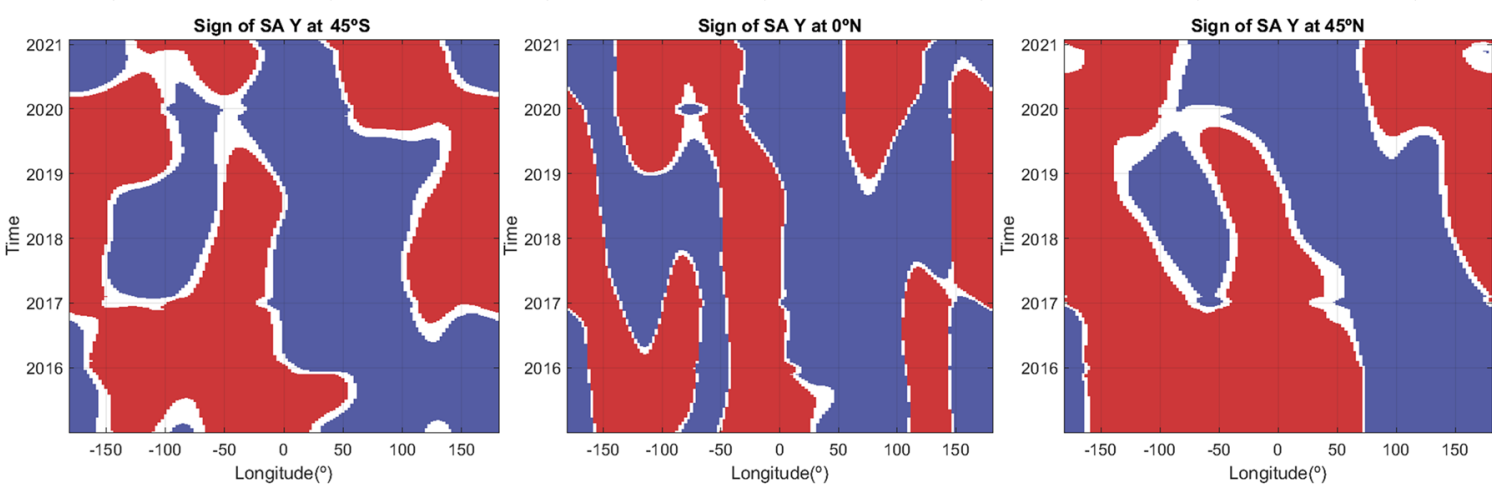

Fig. 3 a SV of the Y-component from CHAOS-7.7 at different locations at the Earth's surface from 2015 to 2021 (a vertical blue line for each year is also plotted). $\mathbf{b}$ Hovmöller diagrams showing the sign of the $S A$ of the $Y$-component at three different latitude profiles ( $45^{\circ} \mathrm{S}$, equator and $45^{\circ} \mathrm{N}$ ). Red colour indicates positive values of SA (increasing trend in the SV), blue indicates negative values of SA (decreasing trend in the SV) and white corresponds to null SA (maximum or minimum in the SV). Yellow stars in a) show the location of the 10 selected observatories labelled by their IAGA code

the 2020-jerk, but also the previous one around 2017. In detail, according to the CHAOS estimations, the 2017jerk shows a clear regional character (Hammer 2018), displaying $\Lambda$-shapes in the Eastern Pacific and CentralNorth American regions around 2017 and V-shapes in the Western Pacific and the Oceania continent. This jerk has not been clearly recorded in the Atlantic region (see Hovmöllers between $50^{\circ} \mathrm{W}$ and $0^{\circ} \mathrm{E}$ for the three latitudinal profiles) and the Indian meridional sector (approx. between $50^{\circ} \mathrm{E}$ and $100^{\circ} \mathrm{E}$ ). The second jerk around 2019-2020 presents a more global character and is practically observed everywhere, except for longitudes between $5^{\circ} \mathrm{E}$ and $50^{\circ} \mathrm{E}$.
To highlight the global evidence of the 2020-jerk, we have explored the changes of the SA of the Y-component at the Earth's surface using the CHAOS model (i.e. main field up to harmonic degree 15). These maps help to identify areas where a jerk shows a higher impact (see Chulliat et al. 2010). The SA changes (labelled here as $\delta \mathrm{SA}$ ) at time $t$ have been estimated as the difference of the SA between $t+6$ months and $t-6$ months (i.e. the SA variation in one year). Maps of the $\delta$ SA of the $Y$-component from August 2015 to June 2020 (given as Additional file 2: Movie S1) show the spatial and temporal evolution of equatorial patches at the Earth's surface that are reinforced during the periods of jerks 

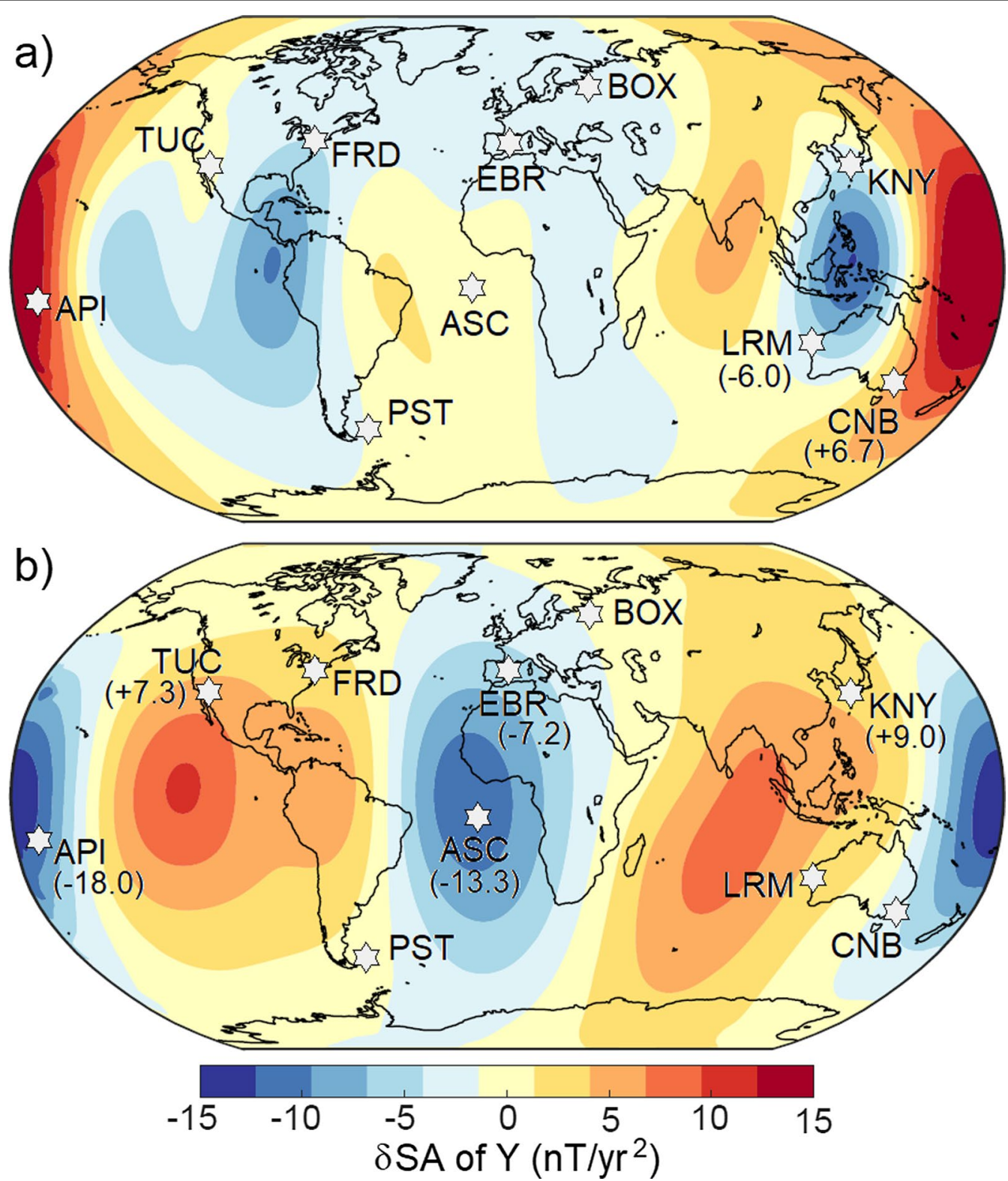

Fig. 4 Maps of the $Y$ secular acceleration changes (SSA) on a July 1st 2017 (2017-jerk) and b May 1st 2019 (2020-jerk) at the Earth's surface obtained from the CHAOS-7.7 model. White stars in both maps indicate the location of the selected observatories. The estimated amplitude of $\delta S A$ from observatory data (Table 1) is also given in parentheses (values in nT/year ${ }^{2}$ ). Amplitudes are not estimated when the jerk was not evident at that observatory or not enough data are available

(Chulliat et al. 2010; Torta et al. 2015). Figure 4 shows two snapshots corresponding to the epochs with the most intense patches during the last 6 years. The first map reflects the 2017-jerk and confirms its regional character with the most intense patches located in the Pacific region. In contrast, the second map shows the global character of the 2020-jerk (the most intense four patches are distributed across the entire equator) where the locations of the previous observatories were plotted to highlight the impact of this jerk on them. Just to benchmark against previous jerk events, a comparison of Fig. 4b with Fig. 3b of Torta et al. (2015) reveals the patchier structure of the 2014-jerk as compared to that of 2020, and a somewhat higher amplitude of the former in global terms. The latter is corroborated by ASC and EBR observatory data (compare the amplitudes given in Table 1 with those from Table 1 in Torta et al. 2015). 
In addition, to compare model estimations and observatory data in terms of $\delta \mathrm{SA}$, we have also shown in Fig. 4 the amplitude of the jerks given by the observatory data (values of Table 1). For the 2020-jerk, the two negative $\delta$ SA patches (covering the Central-Western Pacific Ocean and Western Africa regions) show minimum values in agreement with the $\delta \mathrm{SA}$ amplitude calculated by using the data from API and ASC. Unfortunately, no observatory is located close to the maximum values of the two positive patches (covering the Eastern Pacific and Indian Oceans), although the $\delta$ SA values from TUC and KNY agree with the model estimations in the boundary regions of these patches. This agreement confirms the occurrence of these four patches of $\delta$ SA given by the CHAOS model during the 2020-jerk epoch.

Assuming that geomagnetic jerks are driven by pulses of the secular acceleration of the geomagnetic field at the CMB (Chulliat et al. 2010; Chulliat and Maus 2014; Kloss and Finlay 2019), we have estimated the SA of the radial field $(B r)$ from January 1st 2015 to February 1st 2021 at the CMB. We have calculated $\mathrm{Br}$ in a regular grid of about 10,000 points at the CMB using the CHAOS-7.7. To estimate $\mathrm{Br}$ at the $\mathrm{CMB}$ a maximum harmonic degree of 6 is considered, thus avoiding the larger amplitudes associated with the higher harmonic degrees (Chulliat et al. 2010). To estimate the SA we have calculated the second derivative of $B r$ with $t+6$ months and $t-6$ months.

Figure 5 contains four maps of the SA of $\mathrm{Br}$ (see also Additional file 3: Movie S2) and the spatial global average of the square of SA of $\mathrm{Br}$ (black line in the centre of the figure). Maps show alternating sign lobes close to the equator with more intense values around 2016.0 (Figs. 5a) and mid-2018 (Fig. 5b). In fact, in those epochs the global square average of SA of $\mathrm{Br}$ reaches two maxima or pulses (marked as yellow squares in the black line). This parameter varies substantially over the past 20 years (see Finlay et al. 2015, 2016) and during the last 5 years it has dropped by over $50 \%$ (i.e. from $7 \cdot 10^{4}$ in 2016 to $3 \cdot 10^{4} \mathrm{nT}^{2} /$ year $^{4}$ around mid-2020). These two maps in around 2016.0 and 2018.5 also confirm the anti-correlation (in terms of positive/negative values of SA) between the low-latitude patches in two consecutive pulses related to the existence of equatorial waves propagating at the CMB (Chulliat et al. 2015). After the first pulse (Fig. 5a), the intensity of the patches decreases (Fig. 5c) and a minimum is detected in the global square average of SA during mid-2017 (green square) in agreement with the 2017-jerk. Then, the low-latitude patches reach intense values (Fig. 5b) around mid-2018, moving to low intense values at the end of 2019 where the 2020-jerk occurs (Fig. $5 \mathrm{~d}$ and red square in the blue line). It is important to note that this last map and the trend of the global square average of the SA of $\mathrm{Br}$ close to the red square can be affected by edge effects of the CHAOS model.

Finally, following Torta et al. (2015), we analysed the coherence of the acceleration pulses at the $\mathrm{CMB}$ by means of the power spectra (PS) of the SA of the coefficients of the CHAOS-7.7 model. Additional file 1: Figure $\mathrm{S} 4$ shows the time variation of the SA power of each harmonic degree from 1 (the dipole) to 8 for the last two decades. The peaks, corresponding to the intermediateto-low harmonic degrees, clearly exhibit a 3-year period oscillation with minima during the occurrence of the jerks.

\section{Conclusions}

Although the geomagnetic main field exhibits a complex behaviour in both space and time, whose detailed generating processes remain difficult to establish, recent studies indicate that its small-scale variation is driven by wave oscillations at the core-mantle boundary. These waves suggest a periodic pattern of 3-4 years in the secular acceleration of the field that modulates the occurrence of geomagnetic jerks. Since the last jerk occurred around mid-2017, a new jerk was expected in around 2020. Here, we have confirmed it through a comprehensive study using both ground observatory and satellite data. Our results allow the detection of a new jerk between the end of 2019 and beginning of 2020. In contrast with the regional character of the previous one in 2017, the 2020-jerk is seen more globally according to the observations and CHAOS model estimations. However, care must be taken with this global character due to the limitation of the CHAOS model after mid-2020. Despite the observed SV variation ranges of the Y-component only span around $\pm 5 \mathrm{nT} /$ year in most locations, thanks to the quality of both observatory and Swarm data they are, however, clearly visible. Following the methodology of previous studies, an analysis using a spherical harmonic model of the main field such as CHAOS provides important insights into the origin of this jerk. A new pulse of the secular acceleration of the radial field about mid-2018 at the core-mantle boundary seems to be the trigger of the occurrence of this jerk. Finally, it is worth noting that, similar to what happened with the 2014-jerk, this new jerk occurred soon after the epoch of the latest data available for the production of the IGRF-13 model (Alken et al. 2021), which could affect the predicted secular variation derived from it. However, taking into account the small amplitudes observed in the SA (from both geomagnetic data and CHAOS model), this effect in the IGRF-13 forecast can be minimal. 

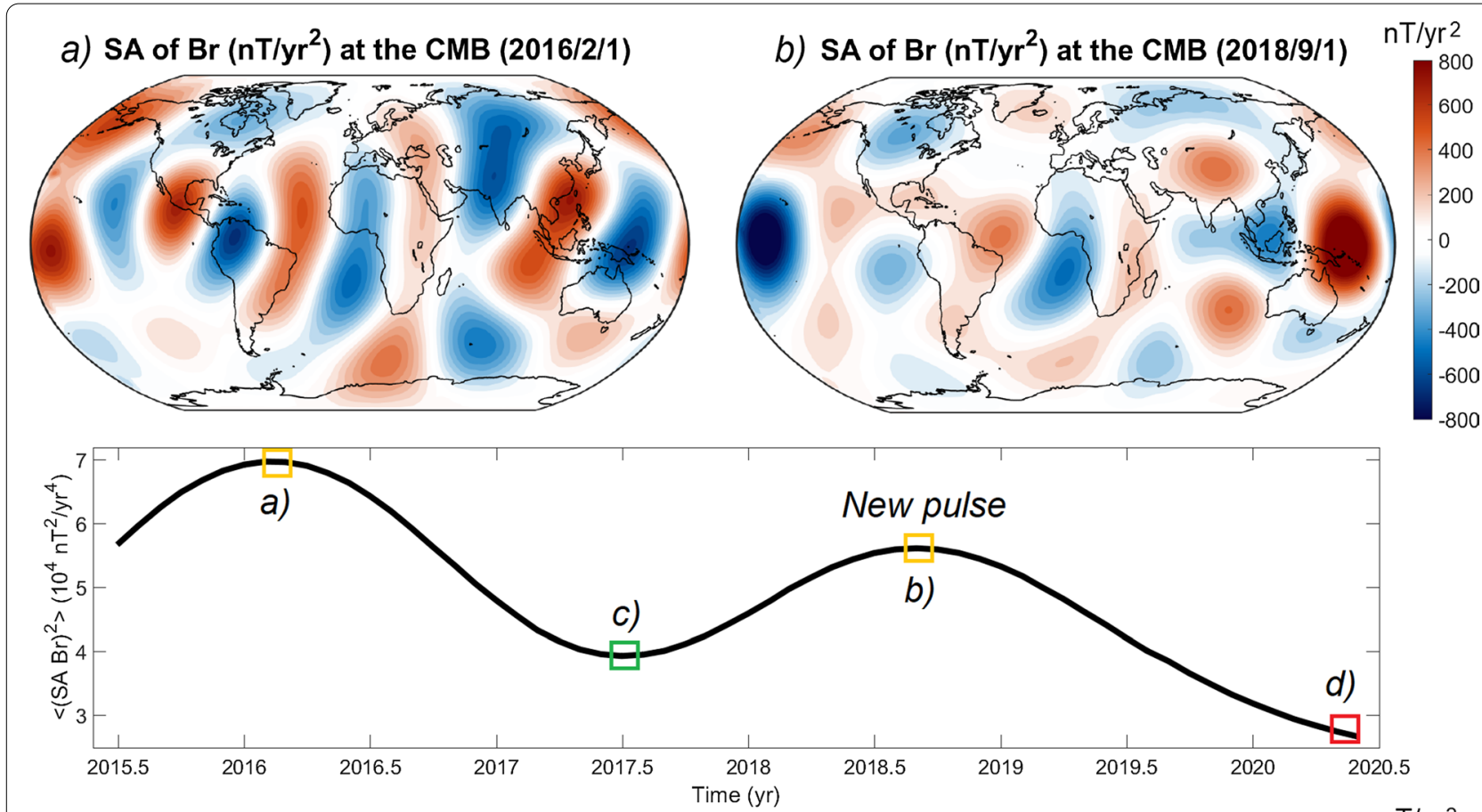

C) SA of $\mathrm{Br}\left(\mathrm{nT} / \mathrm{yr}^{2}\right)$ at the CMB (2017/6/1)

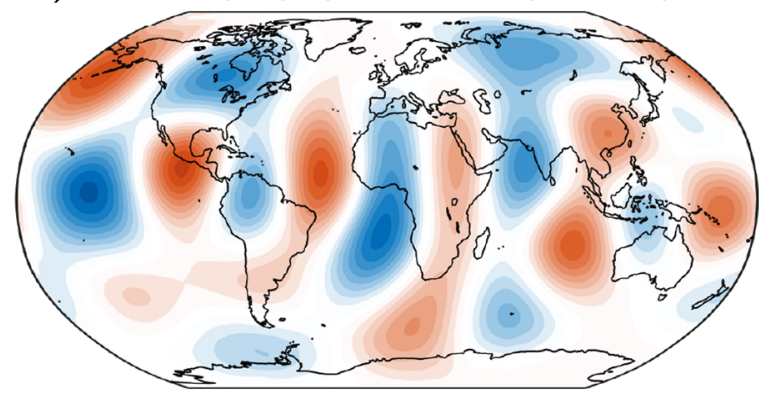

d) $\mathrm{SA}$ of $\mathrm{Br}\left(\mathrm{nT} / \mathrm{yr}^{2}\right)$ at the CMB (2020/6/1)

$\mathrm{nT} / \mathrm{yr}^{2}$

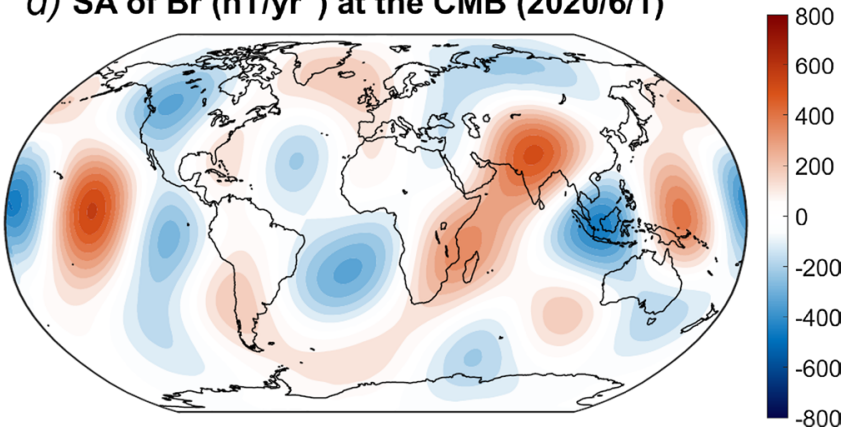

Fig. 5 Maps of the secular acceleration (SA) of the radial field $B r$ at the CMB and the spatial average of its square value given by the black curve. Maps in a February-2016 and b September-2018 correspond to the two maxima observed in the black curve (yellow squares). Map in c June-2017 corresponds to the minimum presented in the black curve (green square) and map in $\mathbf{d}$ June-2020 is plotted at the end of the time window (red square) where the black curve is decreasing

\section{Abbreviations}

CMB: Core-mantle boundary; GVO: Geomagnetic virtual observatories; IAGA: International Association of Geomagnetism and Aeronomy; IGRF: International Geomagnetic Reference Field; PS: Power spectra; SA: Secular acceleration; SV: Secular variation.

\section{Supplementary Information}

The online version contains supplementary material available at https://doi. org/10.1186/s40623-021-01504-2.

Additional file 1. The 2020-jerk in geomagnetic observatory and Swarm data. Figure S1. Monthly means of the East geomagnetic component $Y$ (yellow dots) and the estimations from CHAOS-7.7 model at the same locations (red and blue lines). For an appropriate comparison, the average $Y$ values for the whole time interval have been removed (see text for details). Note that after August 2020 the estimations from CHAOS-7.7 (blue lines) are likely caused by the model regularization instead of the data (see "Discussion: a global view from geomagnetic models" Section of the main text for more details). Figure $\mathbf{S 2}$. Double linear fit of the Y-component SV at geomagnetic observatories leading to the values of Table 1 (see main text). a and $\mathbf{b}$ refer to the 2017-jerk, while $\mathbf{c}$ through $\mathbf{g}$ correspond to the 2020-jerk. Figure S3. Yellow dots: SV of the East component $Y$ from Swarm-A data relocated at $440 \mathrm{~km}$ altitude over the indicated observatory locations (see main text for details). Red and blue curves: SV estimation from CHAOS-7.7 model. Note that after August 2020 the changes in the curves (blue lines) from CHAOS-7.7 are likely caused by the model regularization instead of the data (see "Discussion: a global view from geomagnetic models"Section of the main text for more details).

Figure S4. SA power for each harmonic coefficient from the CHAOS-7.7 model until degree $n=8$ at the CMB. Vertical blue bands indicate the epochs of the reported jerks. Note that after mid-2020 the SA power curves from CHAOS-7.7 are biased by the model parametrization instead of the geomagnetic data providing unreliable values (see "Discussion: a global view from geomagnetic models"Section of the main text for more details). 
Additional file 2. Maps of the $Y$ secular acceleration changes ( $\delta S A$ ) from August 2015 to June 2020 at the Earth's surface obtained from the CHAOS-7.7 model.

Additional file 3. Maps of the secular acceleration (SA) of the radial field at the CMB from July 2015 to June 2020 obtained from the CHAOS-7.7 model.

\section{Acknowledgements}

The authors are grateful to the Spanish research project PGC2018-099103A-100 of the Spanish Ministry of Science, Innovation and Universities. The research that led to these results was also in part carried out using funds from "la Caixa" Foundation. SAC thanks to Juan de la Cierva Formación Program (FJC2018-037643-I). We would like to thank two anonymous reviewers for their constructive comments, which significantly improved our manuscript. This work has used geomagnetic observatory data and we thank all the research institutions and observatories that provide these data, most of them supported by the INTERMAGNET network. We also thank ESA for providing the Swarm Level-1b data.

\section{Authors' contributions}

FJPC and SM compiled, selected and analysed the geomagnetic observatory data and the satellite Swarm data. FJPC and SAC carried out the study with the global model CHAOS. FJPC, SM, SAC and JMT drafted the manuscript. All authors read and approved the final manuscript.

\section{Funding}

This work has been funded by the project PGC2018-099103-A-100 entitled "Candidato Español para Campo Geomagnético de Referencia Internacional 2020" under the umbrella of the programme"I + D de Generación de Conocimiento" of the Spanish Ministry of Science, Innovation and Universities.

\section{Availability of data and materials}

All datasets used in this study can be found in the respective webpages indicated along the manuscript.

\section{Declarations}

Ethics approval and consent to participate

"Not applicable" in this section.

\section{Consent for publication}

"Not applicable" in this section.

\section{Competing interests}

Non-financial competing interests.

\section{Author details}

${ }^{1}$ Universidad Complutense de Madrid (UCM), Plaza Ciencias, 1, 28040 Madrid, Spain. ${ }^{2}$ Observatori de l'Ebre (OE), Univ. Ramon Llull - CSIC, Horta Alta, 38, 43520 Roquetes, Spain. ${ }^{3}$ Geoscience Institute IGEO (CSIC - UCM), C/Doctor Severo Ochoa, 7, 28040 Madrid, Spain.

\section{Received: 31 May 2021 Accepted: 17 August 2021}

Published online: 31 August 2021

\section{References}

Alexandrescu M, Gibert D, Hulot G, Le Mouël J-L, Saracco G (1996) Worldwide wavelet analysis of geomagnetic jerks. J Geophys Res 101:21975-21994. https://doi.org/10.1029/96JB01648

Alken P, Thébault E, Beggan CD, Zhou B et al (2021) International geomagnetic reference field: the thirteenth generation. Earth Planets Space 73(1):1-25. https://doi.org/10.1186/s40623-020-01288-x

Aubert J, Finlay CC (2019) Geomagnetic jerks and rapid hydromagnetic waves focusing at Earth's core surface. Nat Geosci 12(5):393-398
Brown WJ, Mound JE, Livermore PW (2013) Jerks abound: An analysis of geomagnetic observatory data from 1957 to 2008. Phys Earth Planet Inter 223:62-76. https://doi.org/10.1016/j.pepi.2013.06.001

Campuzano SA, Pavón-Carrasco FJ, De Santis A, González-López A, Qamili E (2021) South Atlantic Anomaly areal extent as a possible indicator of geomagnetic jerks in the satellite era. Front Earth Sci 8:607049. https:// doi.org/10.3389/feart.2020.607049

Chulliat A, Maus S (2014) Geomagnetic secular acceleration, jerks, and a localized standing wave at the core surface from 2000 to 2010. J Geophys Res Solid Earth 119:1531-1543. https://doi.org/10.1002/2013JB010604

Chulliat A, Thébault E, Hulot G (2010) Core field acceleration pulse as a common cause of the 2003 and 2007 geomagnetic jerks. Geophys Res Lett 37:L07301. https://doi.org/10.1029/2009GL042019

Chulliat A, Alken P, Maus S (2015) Fast equatorial waves propagating at the top of the Earth's core. Geophys Res Lett 42(9):3321-3329

Duan P, Huang C (2020) Intradecadal variations in length of day and their correspondence with geomagnetic jerks. Nat Commun 11:2273. https://doi. org/10.1038/s41467-020-16109-8

Finlay CC, Olsen N, Tøffner-Clausen L (2015) DTU candidate field models for IGRF-12 and the CHAOS-5 geomagnetic field model. Earth Planets Space 67(1):1-17. https://doi.org/10.1186/s40623-015-0274-3

Finlay CC, Olsen N, Kotsiaros S, Gillet N, Tøffner-Clausen L (2016) Recent geomagnetic secular variation from Swarm and ground observatories as estimated in the CHAOS-6 geomagnetic field model. Earth Planets Space 68(1):1-18. https://doi.org/10.1186/s40623-016-0486-1

Finlay CC, Kloss C, Olsen N, Hammer MD, Tøffner-Clausen L, Grayver A, Kuvshinov A (2020) The CHAOS-7 geomagnetic field model and observed changes in the South Atlantic Anomaly. Earth Planets Space 72(1):1-31. https://doi.org/10.1186/s40623-020-01252-9

Friis-Christensen E, Lühr H, Hulot G (2006) Swarm: a constellation to study the Earth's magnetic field. Earth Planets Space 58:351-358. https://doi.org/10. 1186/bf03351933

Hammer MD, Cox GA, Brown WJ, Beggan CD, Finlay CC (2021) Geomagnetic virtual observatories: monitoring geomagnetic secular variation with the Swarm satellites. Earth Planets Space 73(1):1-22. https://doi.org/10.1186/ s40623-021-01357-9

Hammer, M.D. (2018), Local Estimation of the Earth's Core Magnetic Field. Ph. D. thesis, Technical University of Denmark (DTU). Kgs, Lyngby.

Holme R, De Viron O (2013) Characterization and implications of intradecadal variations in length of day. Nature 499(7457):202-204

Kim HR, von Frese RRB (2013) Localized analysis of polar geomagnetic jerks. Tectonophysics 585:26-33. https://doi.org/10.1016/j.tecto.2012.06.038

Kloss C, Finlay CC (2019) Time-dependent low latitude core flow and geomagnetic field acceleration pulses. Geophys J Int 217(1):140-168

Le Mouël J-L, Ducruix J, Ha Duyen C (1982) The worldwide character of the 1969-1970 impulse of the secular acceleration rate. Phys Earth Planet Inter 28:337-350

Mandea M, Olsen N (2006) A new approach to directly determine the secular variation from magnetic satellite observations. Geophys Res Lett. https:// doi.org/10.1029/2006GL026616

Mandea M, Holme R, Pais A, Pinheiro K, Jackson A, Verbanac G (2010) Geomagnetic jerks: rapid core field variations and core dynamics. Space Sci Rev 155:147-175. https://doi.org/10.1007/s11214-010-9663-x

Olsen N, Mandea M (2007) Investigation of a secular variation impulse using satellite data: the 2003 geomagnetic jerk. Earth Planet Sci Lett 255(1-2):94-105

Olsen N, Mandea M (2008) Rapidly changing flows in the Earth's core. Nat Geosci 1(6):390-394

Olsen N, Mandea M, Sabaka TJ, Tøffner-Clausen L (2009) CHAOS-2-A geomagnetic field model derived from one decade of continuous satellite data. Geophys J Int 179:1477-1487. https://doi.org/10.1111/j.1365-246X.2009. 04386.x

Olsen N, Friis-Christensen E, Floberghagen R, Alken P, Beggan C, Chulliat A, Doornbos E, Encarnacao da Teiexira J, Hamilton B, Hulot G, van den ljssel J, Kuvshinov A, Lesur V, Luehr H, Macmillan S, Maus S, Noja M, Olsen PEH, Park J, Plank G, Puethe C, Ritter P, Rother M, Sabaka TJ, Schachtschneider R, Sirol O, Stolle C, Thébault E, Thomson AWP, Tøffner-Clausen L, Velimsky J, Vigneron P, Visser PN (2013) The swarm satellite constellation 
application and research facility (SCARF) and Swarm data products. Earth Planets Space 65:1189-1200. https://doi.org/10.5047/eps.2013.07.001

Pinheiro KJ, Jackson A, Finlay CC (2011) Measurements and uncertainties of the occurrence time of the 1969,1978, 1991, and 1999 geomagnetic jerks. Geochem Geophys Geosyst 12:Q10015. https://doi.org/10.1029/ 2011 GC003706

Qamili E, De Santis A, Isac A, Mandea M, Duka B, Simonyan A (2013) Geomagnetic jerks as chaotic fluctuations of the Earth's magnetic field. Geochem Geophys Geosyst 14:839-850. https://doi.org/10.1029/2012GC004398

Torta JM, Pavón-Carrasco FJ, Marsal S, Finlay CC (2015) Evidence for a new geomagnetic jerk in 2014. Geophys Res Lett 42:7933-7940. https://doi. org/10.1002/2015GL065501

Wardinski I, Saturnino D, Amit H, Chambodut A, Langlais B, Mandea M,

Thebault E (2020) Geomagnetic core field models and secular variation forecasts for the 13th International Geomagnetic Reference Field (IGRF-13). Earth Planets Space 72(1):1-22. https://doi.org/10.1186/ s40623-020-01254-7

Whaler, K., M. Hammer, C. Finlay, and N. Olsen (2020), Core-mantle boundary flows obtained purely from Swarm secular variation gradient information, In: EGU General Assembly 2020, Online, EGU2020-9616, https://doi.org/ 10.5194/egusphere-egu2020-9616

\section{Publisher's Note}

Springer Nature remains neutral with regard to jurisdictional claims in published maps and institutional affiliations.

\section{Submit your manuscript to a SpringerOpen ${ }^{\circ}$ journal and benefit from:}

- Convenient online submission

- Rigorous peer review

- Open access: articles freely available online

- High visibility within the field

- Retaining the copyright to your article

Submit your next manuscript at $\boldsymbol{\nabla}$ springeropen.com 